\title{
Dynamical susceptibilities of the Falicov-Kimball model with correlated hopping: general approach
}

\author{
A.M.Shvaika, O.Ya.Farenyuk \\ Institute for Condensed Matter Physics of the National Academy of Sciences of Ukraine, \\ 1 Svientsitskii Str., 79011 Lviv, Ukraine
}

Received November 21, 2008, in final form February 18, 2009

The Falicov-Kimball model with correlated hopping is studied in the limit of infinite spatial dimensions. Dynamical susceptibilities are calculated using the generalization of the Dynamical Mean-Field Theory (DMFT), which is based on expansion over electron hopping around the single-site limit. A special case of semi-elliptic density of states and diagonal correlated hopping is considered numerically and the absence of phase transition for all temperatures except $T=0$ is demonstrated, which corresponds to the known results.

Key words: Falicov-Kimball model, correlated hopping, electron susceptibilities, dynamical mean field theory, strong coupling

PACS: $71.10 . \mathrm{Fd}$

\section{Introduction}

The Falicov-Kimball model was first introduced in [1] as a model of metal-insulator transition. It appears that Falicov-Kimball model is the simplest model of electron correlations which displays long-range ordered phases [2]. It was applied to the investigations of such phenomena as valence transitions [3,4], phase separation and crystallization [5]. Dynamical properties of the model, including electron susceptibilities [6,7] and Raman response [8,9] were also investigated. The model contains two types of Fermi-particles: itinerant (conductive) particles, referred to as d-electrons, and localized particles, f-electrons or "ions". On the other hand, the model can be interpreted as an asymmetric Hubbard model with infinite mass of electrons for one of the spin orientations.

Falicov-Kimball model is attractive not only due to its simplicity but also because many exact results were obtained for this model [2]. Among them there are proofs of phase separation for all spatial dimensions of the lattice from one to infinity, theorems of ground-state properties in one and two dimensions. In addition to the results for low spatial dimensions, there exists an exact solution of the model in the infinite dimensions case [10] (see for review [2]). This solution is important in applying Dynamical Mean-Field Theory (DMFT).

DMFT is based on the self-consistent mapping of a lattice problem onto an auxiliary singlesite problem [11]. This transformation is advantageous due to the local nature of self-energy in the limit of infinite spatial dimensions or large coordination. The development of the DMFT approach brought about a noticeable progress in studying strongly correlated systems, but in its traditional form (see, e.g. [11]) it cannot be applied to an important form of hopping, the so-called correlated hopping. Correlated hopping describes the case when the hopping integral depends on the occupation of neighboring sites. Though Falicov-Kimball model gives a reasonable physical description of many processes, it neglects all nonlocal dynamical interactions. Correlated hopping is a notable example of such an interaction. For the first time it was introduced in the well-known Schubin and Wonsowsky work [12], the value of that kind of hopping was estimated for the first time by Hubbard [13], then Hirsch pointed out that correlated hopping can play an important role in the appearance of superconductivity [14]. The importance of the correlated hopping in ferromagnetism was shown in [15-18]. Other reasons for considering the correlated hopping are based on a substantial Coulombian interaction between electrons, reported for some materials [19, 
20], dynamical part of which can be approximated by correlated hopping. When correlated hopping is present, the self-energy becomes non-local, and, as it was shown in earlier investigations [21], this non-locality can be presented as the next-nearest-neighbors hopping contribution to self-energy. But using expansions over electron hopping (Larkin approach, opposite to Dyson approach which is based on expansions over many-electron interactions and leads to the self-energy concept), it can be shown that such non-locality is just an artifact of the Dyson approach. Even for correlated hopping, the irreducible part of Green's function, which cannot be divided by cutting a hopping line, remains local which permits to construct the DMFT for such systems [22].

In this article we develop a general scheme of calculating dynamical susceptibilities for systems with correlated hopping, which is based on generalization of DMFT. It is used to investigate electron susceptibilities of the Falicov-Kimball model with correlated hopping. This method permits to obtain explicit expressions for dynamical susceptibilities. The limit of diagonal correlated hopping on Bethe lattice is studied using the obtained general expressions.

\section{The model}

Hamiltonian of the (spinless) Falicov-Kimball model can be written as:

$$
H=\sum_{i}\left(U n_{i d} n_{i f}-\mu_{\mathrm{f}} n_{i f}-\mu_{\mathrm{d}} n_{i d}\right)+H_{\mathrm{t}}
$$

where $H_{\mathrm{t}}$ is a hopping term for the $\mathrm{d}$-particles, $\mu_{\mathrm{f}}$ and $\mu_{\mathrm{d}}$ are the chemical potentials for the $\mathrm{f}-$ and d-particles, respectively, and $U$ is the Coulomb repulsion of the particles at the same site. Correlated hopping term for the Falicov-Kimball model has the following form:

$$
H_{t}=\frac{1}{\sqrt{D}} \sum_{\langle i, j\rangle}\left[t_{1} d_{i}^{\dagger} d_{j}+t_{2} d_{i}^{\dagger} d_{j}\left(n_{i f}+n_{j f}\right)+t_{3} d_{i}^{\dagger} d_{j} n_{i f} n_{j f}\right] .
$$

Here $t_{1}$ describes the part of hopping between sites $i$ and $j$, which does not depend on the occupation of the sites, $t_{2}$ takes into account the hopping between sites, one of which is occupied by the f-particle, and $t_{3}$ describes the hopping between the sites occupied by the f-particles. Such a Hamiltonian can be presented in terms of hopping between sites with fixed occupations:

$$
\begin{aligned}
H_{t}= & \frac{1}{\sqrt{D}} \sum_{\langle i, j\rangle}\left(t_{i j}^{++} P_{i}^{+} d_{i}^{\dagger} d_{j} P_{j}^{+}+t_{i j}^{--} P_{i}^{-} d_{i}^{\dagger} d_{j} P_{j}^{-}\right. \\
& \left.+t_{i j}^{+-} P_{i}^{+} d_{i}^{\dagger} d_{j} P_{j}^{-}+t_{i j}^{-+} P_{i}^{-} d_{i}^{\dagger} d_{j} P_{j}^{+}\right)=\frac{1}{\sqrt{D}} \sum_{\langle i, j\rangle} \mathbf{d}_{i}^{\dagger} \mathbf{t}_{i j} \mathbf{d}_{j},
\end{aligned}
$$

where $P_{i}^{+}=n_{i f}, P_{i}^{-}=1-n_{i f}$ are the projection operators and we introduce matrix annihilation operator

$$
\mathbf{d}_{i}=\left[\begin{array}{c}
P_{i}^{+} \\
P_{i}^{-}
\end{array}\right] d_{i}
$$

and hopping matrix

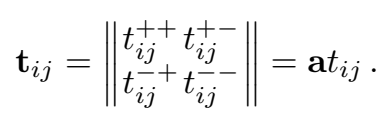

In fact, correlated hopping naturally leads to matrix generalization for all quantities [22]. Both representations for the Hamiltonian are related by the following equations:

$$
\begin{array}{ll}
t^{--}=t_{1}, & t_{1}=t^{--}, \\
t^{+-}=t^{-+}=t_{1}+t_{2}, & t_{2}=t^{+-(-+)}-t^{--}, \\
t^{++}=t_{1}+2 t_{2}+t_{3}, & t_{3}=t^{++}+t^{--}-t^{+-}-t^{-+} .
\end{array}
$$


The components of the matrix Green's function are defined by the following formula:

$$
\begin{aligned}
& G_{i j}^{\alpha \gamma}\left(\tau-\tau^{\prime}\right)=-\left\langle T_{\tau} d_{i \alpha}(\tau) d_{j \gamma}^{\dagger}\left(\tau^{\prime}\right)\right\rangle=\beta \frac{\delta \Omega}{\delta t_{j i}^{\gamma \alpha}\left(\tau^{\prime}-\tau\right)}, \\
& \mathbf{G}_{i j}\left(\tau-\tau^{\prime}\right)=-\left\langle T_{\tau} \mathbf{d}_{i \alpha}(\tau) \otimes \mathbf{d}_{j \gamma}^{\dagger}\left(\tau^{\prime}\right)\right\rangle
\end{aligned}
$$

where $\Omega$ is a grand canonical potential functional. The total Green's function is a sum of all components of the matrix Green's function:

$$
G_{i j}\left(\tau-\tau^{\prime}\right)=-\left\langle T d_{i}(\tau) d_{j}^{+}\left(\tau^{\prime}\right)\right\rangle=\beta \frac{\delta \Omega}{\delta t_{j i}\left(\tau^{\prime}-\tau\right)}=\sum_{\alpha \gamma} G_{i j}^{\alpha \gamma}\left(\tau-\tau^{\prime}\right)
$$

As it was shown in the reference [22], for the systems with correlated hopping, the strongcoupling approach, based on the expansions over electron hopping around the atomic limit, is more convenient. In this approach, the single-electron Green's function is presented as a solution of the matrix generalization of the Larkin equation:

$$
\mathbf{G}_{i j}(\omega)=\boldsymbol{\Xi}_{i j}(\omega)+\sum_{l m} \boldsymbol{\Xi}_{i l}(\omega) \mathbf{t}_{l m} \mathbf{G}_{m j}(\omega),
$$

where $\Xi_{i j}(\omega)$ is an irreducible part of the Green's function which cannot be divided into parts by cutting one hopping line. This irreducible part is local in the limit of infinite spatial dimensions even for the systems with correlated hopping $[22,23]$. After the Fourier transformation to the momentum representation, this equation can be solved for the Green's function:

$$
\mathbf{G}_{\mathbf{k}}(\omega)=\left[\boldsymbol{\Xi}_{\mathbf{k}}^{-1}(\omega)-\mathbf{t}_{\mathbf{k}}\right]^{-1}
$$

with $\mathbf{t}_{\mathbf{k}}=\mathbf{a} \epsilon_{\mathbf{k}}$, where $\epsilon_{\mathbf{k}}$ is an unperturbed band energy. Now we can apply the modified Dynamical Mean Field Theory (DMFT), which leads to exact results in the limit of infinite spatial dimensions $D \rightarrow \infty$, when hopping integrals are scaled according to: $t^{\alpha \beta} \rightarrow t^{\alpha \beta} / \sqrt{D}$. In this limit, an irreducible part is local

$$
\boldsymbol{\Xi}_{\mathbf{k}}(\omega)=\boldsymbol{\Xi}(\omega)
$$

and can be obtained from the condition that single-site Green's function for the lattice and for the auxiliary single-site problem is the same [22]. On the other hand, matrix Green's function of the single-site problem can be written in Larkin representation as:

$$
\mathbf{G}_{\mathrm{imp}}(\omega)=\left[\boldsymbol{\Xi}^{-1}(\omega)-\mathbf{J}(\omega)\right]^{-1}
$$

where $\mathbf{J}$ is the coherent potential matrix ( $\lambda$-field) $[22,10]$. For the Falicov-Kimball model, $\mathbf{G}_{\text {imp }}$ can be calculated exactly:

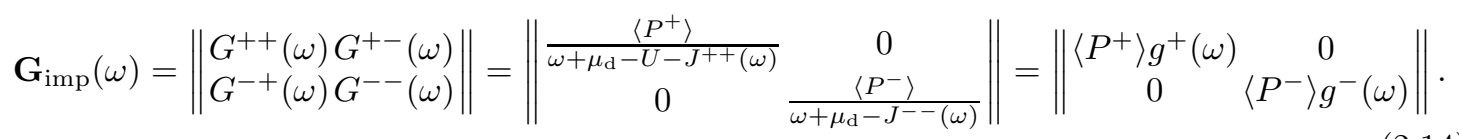

Furthermore, itinerant electrons concentration can be calculated as:

$$
\left\langle n_{\mathrm{d}}\right\rangle=\frac{1}{\beta} \sum_{\nu}\left[G_{\mathrm{imp}}^{++}\left(\mathrm{i} \omega_{\nu}\right)+G_{\mathrm{imp}}^{--}\left(\mathrm{i} \omega_{\nu}\right)\right]=\left\langle P^{+}\right\rangle n_{+}+\left\langle P^{-}\right\rangle n_{-} .
$$

\section{Susceptibilities from the DMFT point of view}

In general, susceptibilities are defined by:

$$
\chi_{i j}^{\mathrm{AB}}\left(\tau-\tau^{\prime}\right)=\left\langle T_{\tau} A_{i}(\tau) B_{j}\left(\tau^{\prime}\right)\right\rangle .
$$


For instance, itinerant electron charge susceptibilities for the Falicov-Kimball model with correlated hopping can be obtained from the following matrix of projected susceptibilities:

$$
\chi_{i j}^{n n}\left(\tau-\tau^{\prime}\right)=\left\|\begin{array}{ll}
\left\langle T_{\tau} P_{i}^{+} n_{i d}(\tau) n_{j d}\left(\tau^{\prime}\right) P_{j}^{+}\right\rangle & \left\langle T_{\tau} P_{i}^{+} n_{i d}(\tau) n_{j d}\left(\tau^{\prime}\right) P_{j}^{-}\right\rangle \\
\left\langle T_{\tau} P_{i}^{-} n_{i d}(\tau) n_{j d}\left(\tau^{\prime}\right) P_{j}^{+}\right\rangle & \left\langle T_{\tau} P_{i}^{-} n_{i d}(\tau) n_{j d}\left(\tau^{\prime}\right) P_{j}^{-}\right\rangle
\end{array}\right\|
$$

Analyzing the diagrammatical series, obtained by using Wick theorem in the same way as in [7], one can see that many-particle Green functions can be written in the following form:

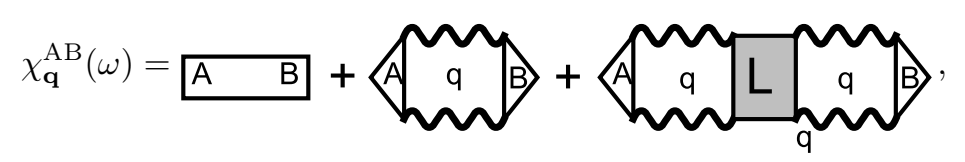

where

$$
\tilde{t}_{\mathrm{q}}=m=m+\sim \sim m
$$

is a sum of the chains of hopping and arrow denotes lattice Green's function (2.11). Here and $L$ are irreducible parts that cannot be divided by cutting two hopping lines and

$$
\sim_{\sim}^{\mathrm{q}}=-\frac{1}{N} \sum_{k} \tilde{\mathbf{t}}_{k} \otimes \tilde{\mathbf{t}}_{k+q}
$$

It should be noted, that here correlated hopping leads to a Cartesian product over $\alpha=+$ and - in place of ordinary multiplication in the similar equations for systems without correlated hopping [7]. The full four-vertex in equation (3.3) is connected with the irreducible one $L$ by the BetheSalpeter-like equation:

$$
L_{q}=L+L L_{q}^{q} L_{q}
$$

All the introduced irreducible parts are local in the limit of infinite spatial dimensions [7] and can be calculated from the corresponding single-site many-particle Green functions:

$\Upsilon\left(\begin{array}{ll}1 & 4 \\ 2 & 3\end{array}\right)=\bigcup_{2}^{1} \bigcup_{3}^{4} \equiv\left\langle T_{\tau} a_{1}^{\dagger} a_{2} a_{3}^{\dagger} a_{4}\right\rangle_{\mathrm{imp}}-\left\langle T_{\tau} a_{1}^{\dagger} a_{2}\right\rangle_{\mathrm{imp}}\left\langle T_{\tau} a_{3}^{\dagger} a_{4}\right\rangle_{\mathrm{imp}}+\left\langle T_{\tau} a_{1}^{\dagger} a_{4}\right\rangle_{\mathrm{imp}}\left\langle T_{\tau} a_{3}^{\dagger} a_{2}\right\rangle_{\mathrm{imp}}$,

where $\Upsilon$ is $4 \times 4$ matrix, whose indices take the values "++", "--", "+-", "-+";

$$
\begin{aligned}
& 0\left\langle{ }_{2}^{1} \equiv\left\langle T_{\tau} \hat{A}_{0} a_{1}^{\dagger} a_{2}\right\rangle_{\mathrm{imp}}-\left\langle\hat{A}_{0}\right\rangle_{\mathrm{imp}}\left\langle T_{\tau} a_{1}^{\dagger} a_{2}\right\rangle_{\mathrm{imp}},\right. \\
& 0 \mathrm{~A} \quad \mathrm{~B} 1 \equiv\left\langle T_{\tau} \hat{A}_{0} \hat{B}_{1}\right\rangle_{\mathrm{imp}}-\left\langle\hat{A}_{0}\right\rangle_{\mathrm{imp}}\left\langle\hat{B}_{1}\right\rangle_{\mathrm{imp}} .
\end{aligned}
$$

Quantity (3.7) correspond to the following diagrammatical sequence:

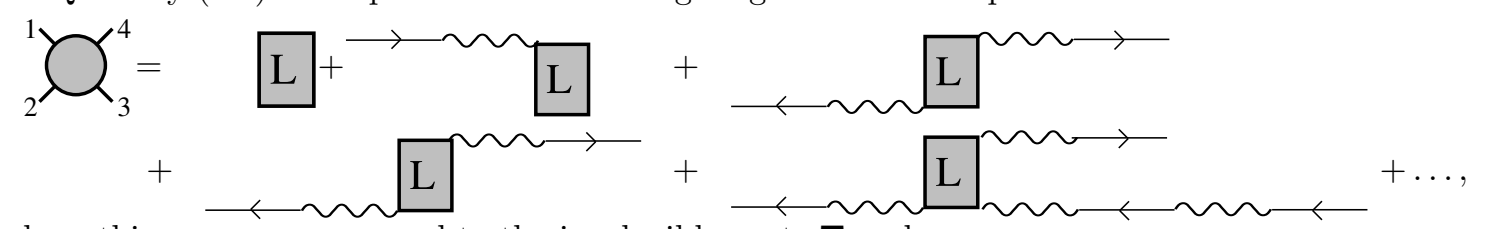

where thin arrows correspond to the irreducible parts $\boldsymbol{\Xi}$ and

$$
L=L+L C L
$$


Now, using definition (3.4), one can state that:

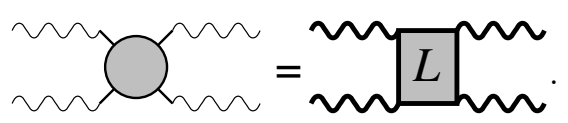

Here the thin wavy lines correspond to the coherent potential $\mathbf{J}$, the thick one

$$
\tilde{\mathbf{J}}=\boldsymbol{m}=\varkappa+\sim \longrightarrow \sim
$$

is the sum of chains of the wavy lines, and a line above another one should be treated as [see also (3.5)]:

$$
\sim^{\prime}=-\tilde{\mathbf{J}} \otimes \tilde{\mathbf{J}}
$$

From equations (3.10) and (3.6) the four-vertex $\mathrm{L}_{\mathrm{q}}$ can be obtained as

$$
L=\left[L{ }^{-1}+m^{-} \sim_{q}^{q}\right]^{-1} .
$$

In the same way, equations (3.8) and (3.9) for other irreducible many-particle Green functions can be written down:

$$
\begin{aligned}
& \Omega^{m}=m^{m}+m L \\
& A B=A B D-A B D-A
\end{aligned}
$$

After performing all the necessary substitutions into equation (3.3) one can obtain the following formula for the lattice susceptibility $\chi_{\mathbf{q}}^{\mathrm{AB}}\left(\omega_{n}\right)$ :

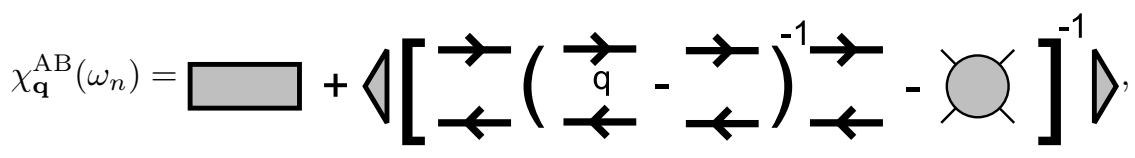

where

$$
\begin{aligned}
& \stackrel{+}{\leftarrow}=-\mathbf{G}_{\mathrm{imp}} \otimes \mathbf{G}_{\mathrm{imp}} \\
& \underset{\stackrel{\mathbf{q}}{\leftarrow}}{\leftarrow}=-\frac{1}{N} \sum_{\mathbf{k}} \mathbf{G}_{\sigma \mathbf{k}}\left(\omega_{\nu}\right) \otimes \mathbf{G}_{\sigma \mathbf{k}+\mathbf{q}}\left(\omega_{\nu+m}\right) .
\end{aligned}
$$

In equation (3.17), quantity $\Lambda=[\ldots]^{-1}$ denotes an inverse kernel of the corresponding integral equation:

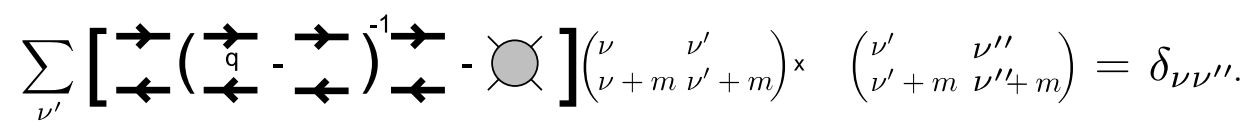

Many-particle correlation function (3.7) contains two contributions (see Appendix):

$$
\Upsilon=\left\|\begin{array}{cc}
\boldsymbol{\Upsilon}_{s}^{1} & 0 \\
0 & \mathbf{\Upsilon}_{s}^{2}
\end{array}\right\| \delta_{\nu \nu^{\prime}}-\left\|\begin{array}{cc}
\boldsymbol{\Upsilon}_{\mathrm{d}}^{1} 0 \\
0 & 0
\end{array}\right\| \delta_{m 0}
$$


As a result, the expression enclosed in square brackets in (3.20) consists of the terms diagonal on internal $\nu=\nu^{\prime}$ and external $\nu=\nu+m(m=0)$ frequencies too:

$$
[\ldots]=\boldsymbol{\Pi}\left(\begin{array}{cc}
\nu & \nu^{\prime} \\
\nu+m & \nu^{\prime}+m
\end{array}\right) \delta_{\nu \nu^{\prime}}-\left\|\begin{array}{cc}
\mathbf{\Upsilon}_{\mathrm{d}}^{1} 0 \\
0 & 0
\end{array}\right\|\left(\begin{array}{cc}
\nu & \nu^{\prime} \\
\nu+m \nu^{\prime}+m
\end{array}\right) \delta_{m 0} .
$$

The term, diagonal on internal frequencies is equal to:

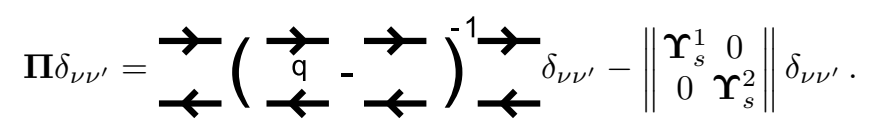

Explicit form of irreducible many-particle Green functions of the single-site model is presented in the Appendix.

Since the matrix for the 3-time irreducible Green's function has the following structure:

$$
\downarrow=\left[\downarrow_{1}, \mathbf{0}\right]
$$

and is constructed by the $2 \times 2$ blocks (see Appendix), we are interested only in the upper left $2 \times 2$ block of matrix $\Lambda$ :

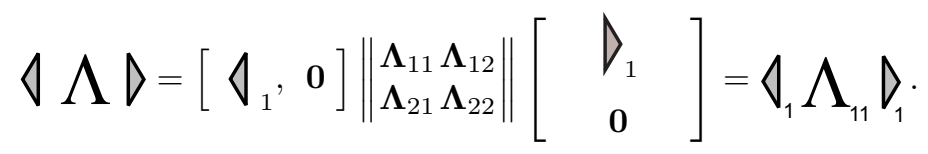

Substituting (3.22) and (3.23) into (3.20) one can obtain:

$$
\begin{aligned}
& \sum_{\nu^{\prime}}\left[\| \begin{array}{l}
\boldsymbol{\Pi}_{11} \boldsymbol{\Pi}_{12} \\
\boldsymbol{\Pi}_{21} \boldsymbol{\Pi}_{22}\left\|\left(\begin{array}{ll}
\nu & \nu^{\prime} \\
\nu+m & \nu^{\prime}+m
\end{array}\right) \delta_{\nu \nu^{\prime}}-\left\langle P^{+}\right\rangle\left\langle P^{-}\right\rangle\right\|\left[\begin{array}{l}
g_{\nu}^{+} \\
g_{\nu}^{-}
\end{array}\right] \otimes\left[\begin{array}{c}
g_{\nu^{\prime}}^{+}, g_{\nu^{\prime}}^{-} \\
0
\end{array}\right] \| \delta_{m 0} \\
0
\end{array}\right] \\
& \times\left\|\begin{array}{ll}
\boldsymbol{\Lambda}_{11} \boldsymbol{\Lambda}_{12} \\
\boldsymbol{\Lambda}_{21} & \boldsymbol{\Lambda}_{22}
\end{array}\right\|\left(\begin{array}{ll}
\nu^{\prime} & \nu^{\prime \prime} \\
\nu^{\prime}+m & \nu^{\prime \prime}+m
\end{array}\right)=\delta_{\nu \nu^{\prime \prime}} \mathrm{I}^{(4)},
\end{aligned}
$$

which leads to the following closed system of equations for $\boldsymbol{\Lambda}_{11}$ and $\boldsymbol{\Lambda}_{21}$ :

$$
\begin{aligned}
& \boldsymbol{\Pi}_{11}\left(\begin{array}{c}
\nu \nu \\
\nu \nu
\end{array}\right) \boldsymbol{\Lambda}_{11}\left(\begin{array}{c}
\nu \nu^{\prime \prime} \\
\nu \nu^{\prime \prime}
\end{array}\right)+\boldsymbol{\Pi}_{12}\left(\begin{array}{c}
\nu \nu \\
\nu \nu
\end{array}\right) \boldsymbol{\Lambda}_{21}\left(\begin{array}{l}
\nu \nu^{\prime \prime} \\
\nu \nu^{\prime \prime}
\end{array}\right) \\
& -\delta_{m 0}\left\langle P^{+}\right\rangle\left\langle P^{-}\right\rangle \sum_{\nu^{\prime}}\left\{\left[g_{\nu}^{+} g_{\nu}^{-}\right] \otimes\left[\begin{array}{c}
g_{\nu^{\prime}}^{+} \\
g_{\nu^{\prime}}^{-}
\end{array}\right]\right\} \boldsymbol{\Lambda}_{11}\left(\begin{array}{c}
\nu^{\prime} \nu^{\prime \prime} \\
\nu^{\prime} \nu^{\prime \prime}
\end{array}\right)=\delta_{\nu \nu^{\prime \prime}} \mathrm{I}^{(2)}, \\
& \boldsymbol{\Pi}_{21}\left(\begin{array}{c}
\nu \nu \\
\nu \nu
\end{array}\right) \boldsymbol{\Lambda}_{11}\left(\begin{array}{c}
\nu \nu^{\prime \prime} \\
\nu \nu^{\prime \prime}
\end{array}\right)+\Pi_{22}\left(\begin{array}{c}
\nu \nu \\
\nu \nu
\end{array}\right) \boldsymbol{\Lambda}_{21}\left(\begin{array}{c}
\nu \nu^{\prime \prime} \\
\nu \nu^{\prime \prime}
\end{array}\right)=0 .
\end{aligned}
$$

Matrix $\boldsymbol{\Lambda}_{21}$ can be obtained from the equation (3.28). After its substitution into the formula (3.27), one obtains final system of equations. This system is algebraic for the dynamic susceptibilities when $m \neq 0$. For the static susceptibilities $m=0$ one has a system of integral equations with separable kernel, which can be easily solved by multiplicating both sides of equation from the left by $\left[g_{\nu}^{+}, g_{\nu}^{-}\right]$ and carrying out the sum over frequency $\nu$ :

$$
\frac{1}{\beta} \sum_{\nu}\left[g_{\nu}^{+} g_{\nu}^{-}\right] \boldsymbol{\Lambda}_{11}\left(\begin{array}{cc}
\nu & \nu^{\prime \prime} \\
\nu & \nu^{\prime \prime}
\end{array}\right)=\frac{\left[g_{\nu^{\prime \prime}}^{+}, g_{\nu^{\prime \prime}}^{-}\right] \mathbf{D}_{11}^{-1}\left(\nu^{\prime \prime}\right)}{T-\Theta}
$$

where:

$$
\mathbf{D}_{11}(\nu)=\boldsymbol{\Pi}_{11}\left(\begin{array}{cc}
\nu & \nu \\
\nu & \nu
\end{array}\right)-\boldsymbol{\Pi}_{12}\left(\begin{array}{cc}
\nu & \nu \\
\nu & \nu
\end{array}\right) \boldsymbol{\Pi}_{22}^{-1}\left(\begin{array}{ll}
\nu & \nu \\
\nu & \nu
\end{array}\right) \boldsymbol{\Pi}_{21}\left(\begin{array}{ll}
\nu & \nu \\
\nu & \nu
\end{array}\right)
$$

and

$$
\Theta=\left\langle P^{+}\right\rangle\left\langle P^{-}\right\rangle \frac{1}{\beta} \sum_{\nu}\left[\begin{array}{l}
g_{\nu}^{+} \\
g_{\nu}^{-}
\end{array}\right] D_{11}^{-1}(\nu)\left[g_{\nu}^{+}, g_{\nu}^{-}\right]
$$


determines critical temperature by equation $T-\Theta(T, q)=0$.

Finally one can find

$$
\boldsymbol{\Lambda}_{11}\left(\begin{array}{cc}
\nu & \nu^{\prime \prime} \\
\nu & \nu^{\prime \prime}
\end{array}\right)=\mathbf{D}_{11}^{-1}(\nu) \delta_{\nu \nu^{\prime \prime}}+\frac{\left\langle P^{+}\right\rangle\left\langle P^{-}\right\rangle}{T-\Theta} \mathbf{D}_{11}^{-1}(\nu)\left[\begin{array}{l}
g_{\nu}^{+} \\
g_{\nu}^{-}
\end{array}\right] \otimes\left[\begin{array}{ll}
g_{\nu^{\prime \prime}}^{+} & g_{\nu^{\prime \prime}}^{-}
\end{array}\right] \mathbf{D}_{11}^{-1}\left(\nu^{\prime \prime}\right)
$$

which must be substituted in equation (3.25) and then in (3.17).

\section{Diagonal correlated hopping in the case of semi-elliptic density of states}

Equation (3.25) together with (3.17) gives an exact analytical form for the electron susceptibilities, but to rewrite this equation explicitly one need to calculate the inverse of the $4 \times 4$ matrix in (3.23). Although, this can be easily done, the resulting expressions are cumbersome. In order to proceed, we can consider a simpler case, when correlated hopping and coherent potential are both diagonal $\left(a^{+-}=a^{-+}=0\right)$. For the Bethe lattice with semielliptic density of states

$$
\rho(t)=\frac{2}{\pi W^{2}} \sqrt{W^{2}-t^{2}}
$$

DMFT system of equations is reduced to the matrix one [22]:

$$
\mathbf{J}(\omega)=\frac{W^{2}}{4} \mathbf{a G}_{\text {imp }}(\omega) \mathbf{a} .
$$

Solving equation (4.2) for diagonal correlated hopping, one can obtain expressions for the coherent potential [22]:

$$
\begin{aligned}
& J^{++}(\omega)=\frac{\left(a^{++}\right)^{2} W^{2}}{4} G^{++}(\omega)=\frac{1}{2}\left[\omega+\mu_{\mathrm{d}}-U \pm \mathrm{i} \sqrt{W_{+}^{2}-\left(\omega+\mu_{\mathrm{d}}-U\right)^{2}}\right], \\
& J^{--}(\omega)=\frac{\left(a^{--}\right)^{2} W^{2}}{4} G^{--}(\omega)=\frac{1}{2}\left[\omega+\mu_{\mathrm{d}} \pm \mathrm{i} \sqrt{W_{-}^{2}-\left(\omega+\mu_{\mathrm{d}}\right)^{2}}\right], \\
& J^{+-}(\omega)=0, \\
& J^{-+}(\omega)=0,
\end{aligned}
$$

where: $W_{ \pm}^{2}=\left(a^{ \pm \pm}\right)^{2} W^{2}\left\langle P^{ \pm}\right\rangle$. Lattice Green functions also acquire a simple form:

$$
\begin{aligned}
G_{\mathbf{k}}^{++} & =\frac{\left\langle P^{+}\right\rangle}{\omega+\mu_{\mathrm{d}}-U-J^{++}\left\langle P^{-}\right\rangle-a^{++}\left\langle P^{+}\right\rangle \epsilon_{\mathbf{k}}}, \\
G_{\mathbf{k}}^{--} & =\frac{\left\langle P^{-}\right\rangle}{\omega+\mu_{\mathrm{d}}-U-J^{--}\left\langle P^{+}\right\rangle-a_{k}^{--}\left\langle P^{-}\right\rangle \epsilon_{\mathbf{k}}} .
\end{aligned}
$$

As a result, matrices $\mathbf{W}$ and $\mathbf{D}_{11}$ will be diagonal and $\Theta(T, q)$ will be equal to:

$$
\Theta(T, \mathbf{q})=\left\langle P^{+}\right\rangle\left\langle P^{-}\right\rangle \beta \sum_{\nu}\left[g_{\nu}^{+} \boldsymbol{\Pi}_{++,++}^{-1}(\nu, \mathbf{q}) g_{\nu}^{+}+g_{\nu}^{-} \boldsymbol{\Pi}_{--,--}^{-1}(\nu, \mathbf{q}) g_{\nu}^{-}\right],
$$

where

$$
\boldsymbol{\Pi}_{\alpha \alpha, \alpha \alpha}(\nu, \mathbf{q})=g_{\nu}^{\alpha} g_{\nu}^{\alpha} \frac{G_{\nu}^{\alpha \alpha} G_{\nu}^{\alpha \alpha}-\frac{1}{N} \sum_{\mathbf{k}} G_{\nu, \mathbf{k}}^{\alpha \alpha} G_{\nu, \mathbf{k}+\mathbf{q}}^{\alpha \alpha}}{G_{\nu}^{\alpha \alpha} G_{\nu}^{\alpha \alpha}\left[2\left\langle P^{\alpha}\right\rangle-1\right]+\left\langle P^{\alpha}\right\rangle \frac{1}{N} \sum_{\mathbf{k}} G_{\nu, \mathbf{k}}^{\alpha \alpha} G_{\nu, \mathbf{k}+\mathbf{q}}^{\alpha \alpha}}=g_{\nu}^{\alpha} g_{\nu}^{\alpha}\left[L_{\nu, \mathbf{q}}^{\alpha \alpha}\right]^{-1}
$$

which leads to:

$$
\Theta(T, \mathbf{q})=\left\langle P^{+}\right\rangle\left\langle P^{-}\right\rangle \frac{1}{\beta} \sum_{\nu}\left(L_{\nu, \mathbf{q}}^{++}+L_{\nu, \mathbf{q}}^{--}\right)
$$


In the case of the Bethe lattice (Kelly-tree) the sum over $\mathbf{k}$ cannot be correctly defined in general. But we can redefine it for the uniform $(\mathbf{q}=0)$ and chess-board $[\mathbf{q}=(\pi, \pi, \pi, \ldots)]$ responses. When correlated hopping is diagonal, such a sum can be easily calculated:

$$
\frac{1}{N} \sum_{\mathbf{k}} G_{\mathbf{k}, \nu}^{\alpha \alpha} G_{\mathbf{k}+\mathbf{q}, \nu}^{\alpha \alpha}=\left\{\begin{array}{l}
\frac{1}{N} \sum_{\mathbf{k}} G_{\mathbf{k}, \nu}^{\alpha \alpha} G_{\mathbf{k}, \nu}^{\alpha \alpha}=\int d t \rho(t)\left[\Xi_{\nu}^{-1}-a^{\alpha \alpha}\left\langle P^{\alpha}\right\rangle t\right]^{-1}\left[\Xi_{\nu}^{-1}-a^{\alpha \alpha}\left\langle P^{\alpha}\right\rangle t\right]^{-1}, q=0 \\
\frac{1}{N} \sum_{\mathbf{k}} G_{\mathbf{k}, \nu}^{\alpha \alpha} G_{-\mathbf{k}, \nu}^{\alpha \alpha}=\int d t \rho(t)\left[\Xi_{\nu}^{-1}-a^{\alpha \alpha}\left\langle P^{\alpha}\right\rangle t\right]^{-1}\left[\Xi_{\nu}^{-1}+a^{\alpha \alpha}\left\langle P^{\alpha}\right\rangle t\right]^{-1}, q=\pi
\end{array}\right.
$$

which gives

$$
\frac{1}{N} \sum_{k} G_{\mathbf{k}, \nu}^{\alpha \alpha} G_{\mathbf{k}+\mathbf{q}, \nu}^{\alpha \alpha}= \begin{cases}\frac{-G_{\nu}^{\alpha \alpha} G_{\nu}^{\alpha \alpha}}{1-G_{\nu}^{\alpha \alpha} J_{\nu}^{\alpha \alpha}} & q=0 \\ \frac{+G_{\nu}^{\alpha \alpha} G_{\nu}^{\alpha \alpha}}{1+G_{\nu}^{\alpha \alpha} J_{\nu}^{\alpha \alpha}}, & q=\pi\end{cases}
$$

and

$$
L_{\nu, \mathbf{q}}^{\alpha \alpha}=\frac{ \pm G_{\nu}^{\alpha \alpha} J_{\nu}^{\alpha \alpha}}{\left\langle P^{\alpha}\right\rangle \pm\left(2\left\langle P^{\alpha}\right\rangle-1\right) G_{\nu}^{\alpha \alpha} J_{\nu}^{\alpha \alpha}}
$$

where "-" $\operatorname{sign}$ corresponds to $\mathbf{q}=0$ and "+" $\operatorname{sign}$ to $\mathbf{q}=(\pi, \pi, \pi, \ldots)$. As a result:

$$
\begin{aligned}
& \chi_{q}^{\mathrm{AB}}(m=0)=\mathrm{A} \quad \mathrm{B}+\sum_{\nu}\left\{\left\|\begin{array}{cc}
L_{\nu}^{++} g_{\nu}^{+} g_{\nu}^{+}\left\langle P^{+}\right\rangle & 0 \\
0 & L_{\nu}^{--} g_{\nu}^{-} g_{\nu}^{-}\left\langle P^{-}\right\rangle
\end{array}\right\|+\left\langle P^{+}\right\rangle\left\langle P^{-}\right\rangle\left[\begin{array}{c}
L_{\nu}^{++} n_{+} \\
-L_{\nu}^{--} n_{-}
\end{array}\right]\right. \\
& \otimes\left[g_{\nu}^{+},-g_{\nu}^{-}\right]+\left\langle P^{+}\right\rangle\left\langle P^{-}\right\rangle\left[\begin{array}{c}
n_{+} \\
-n_{-}
\end{array}\right] \otimes\left[L_{\nu}^{++} g_{\nu}^{+},-L_{\nu}^{--} g_{\nu}^{-}\right]+\left[\begin{array}{c}
n_{+} \\
-n_{-}
\end{array}\right] \\
& \left.\otimes\left[n_{+} L_{\nu}^{++}\left\langle P^{-}\right\rangle,-n_{-} L_{\nu}^{--}\left\langle P^{+}\right\rangle\right]+\left[\begin{array}{c}
n_{+} \\
-n_{-}
\end{array}\right] \otimes\left[n_{-} g_{\nu}^{+}\left(g_{\nu}^{-}\right)^{-1} L_{\nu}^{--}\left\langle P^{+}\right\rangle,-n_{+} g_{\nu}^{-}\left(g_{\nu}^{+}\right)^{-1} L_{\nu}^{++}\left\langle P^{-}\right\rangle\right]\right\} \\
& +\frac{\left\langle P^{+}\right\rangle\left\langle P^{-}\right\rangle}{T-\Theta(T, q)} \sum_{\nu \nu^{\prime}}\left\{\left[\begin{array}{l}
L_{\nu}^{++} g_{\nu}^{+} \\
L_{\nu}^{--} g_{\nu}^{-}
\end{array}\right] \otimes\left[L_{\nu^{\prime}}^{++} g_{\nu^{\prime}}^{+}, L_{\nu^{\prime}}^{--} g_{\nu^{\prime}}^{-}\right]+\left[\begin{array}{l}
L_{\nu}^{++} g_{\nu}^{+} \\
L_{\nu}^{--} g_{\nu}^{-}
\end{array}\right] \otimes\left[n_{+} L_{\nu^{\prime}}^{++}\left\langle P^{-}\right\rangle, n_{-} L_{\nu^{\prime}}^{--}\left\langle P^{+}\right\rangle\right]\right. \\
& -\left[\begin{array}{l}
L_{\nu}^{++} g_{\nu}^{+} \\
L_{\nu}^{-}-g_{\nu}^{-}
\end{array}\right] \otimes\left[n_{-} g_{\nu^{\prime}}^{+}\left(g_{\nu^{\prime}}^{-}\right)^{-1} L_{\nu^{\prime}}^{--}\left\langle P^{+}\right\rangle, n_{+} g_{\nu^{\prime}}^{-}\left(g_{\nu^{\prime}}^{+}\right)^{-1} L_{\nu^{\prime}}^{++}\left\langle P^{-}\right\rangle\right] \\
& +\left(L_{\nu}^{++}\left\langle P^{-}\right\rangle-L_{\nu}^{--}\left\langle P^{+}\right\rangle\right)\left[\begin{array}{c}
n_{+} \\
-n_{-}
\end{array}\right] \otimes\left[L_{\nu^{\prime}}^{++} g_{\nu^{\prime}}^{+}, L_{\nu^{\prime}}^{--} g_{\nu^{\prime}}^{-}\right]+\left(L_{\nu}^{++}\left\langle P^{-}\right\rangle-L_{\nu}^{--}\left\langle P^{+}\right\rangle\right)\left[\begin{array}{c}
n_{+} \\
-n_{-}
\end{array}\right] \\
& \otimes\left[n_{+} L_{\nu^{\prime}}^{++}\left\langle P^{-}\right\rangle, n_{-} L_{\nu^{\prime}}^{--}\left\langle P^{+}\right\rangle\right]-\left(L_{\nu}^{++}\left\langle P^{-}\right\rangle-L_{\nu}^{--}\left\langle P^{+}\right\rangle\right)\left[\begin{array}{c}
n_{+} \\
-n_{-}
\end{array}\right] \\
& \left.\otimes\left[n_{-} g_{\nu^{\prime}}^{+}\left(g_{\nu^{\prime}}^{-}\right)^{-1} L_{\nu^{\prime}}^{--}\left\langle P^{+}\right\rangle, n_{+} g_{\nu^{\prime}}^{-}\left(g_{\nu^{\prime}}^{+}\right)^{-1} L_{\nu^{\prime}}^{++}\left\langle P^{-}\right\rangle\right]\right\} \text {. }
\end{aligned}
$$

Critical temperature is determined by the divergence of susceptibility and can be obtained from the equation $T-\Theta(T, \mathbf{q})=0$. The solutions of this equation were studied numerically and revealed that it can have only one solution $T=0$ and the absence of phase transition for all temperatures except $T=0$ can be stated. That result conforms with the known results for binary alloys with offdiagonal randomness [24,25]. Physical reason for that is the absence of self-intersections in Bethe lattice along with diagonal correlated hopping. In our case diagonal correlated hopping means that hopping is allowed only between sites with the same occupation of f-particles. That leads the lattice to "break-down" into finite-sized domains of sites with the same f-particles occupation "diluted" by the domains of sites of the other occupation.

It should be noted that in the case of a fixed total electron concentration $n_{\mathrm{d}}+n_{\mathrm{f}}=$ const the phase transition into a mixed-valence phase can take place in the Falicov-Kimball model without divergence of charge susceptibilities [26]. Such transitions can take place only when the energy level of f-states $E_{\mathrm{f}}=\mu_{\mathrm{d}}-\mu_{\mathrm{f}}$ is negative $E_{\mathrm{f}}<0$ which is not the case considered in the current article. 


\section{Conclusions}

In this paper we have investigated the susceptibilities of the Falicov-Kimball model with correlate hopping. The model was studied using modified DMFT, applicable to systems with correlated hopping. Diagrammatical expression for the susceptibilities was obtained and proved to be identical to the corresponding diagrammatic expression for the ordinary Falicov-Kimball model. Starting from this expression, explicit formulas for susceptibilities were obtained.

As a particular case we have studied electron charge susceptibilities for the case of Bethe lattice with correlated hopping, which corresponds to the hopping only between sites with the same occupation. It was shown that phase transition cannot occur in such a system for any nonzero temperature which is consistent with the earlier result in the CPA approach. 


\section{Appendix. Irreducible many-particle Green functions for the Falicov-Kimball model}

Four-time Green's function contains only the following non-zero components:

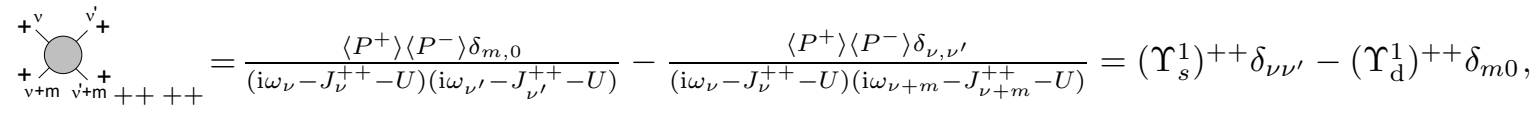

$$
\begin{aligned}
& -_{v+m}^{n^{\prime}+m_{----}^{-}} v_{-}^{v^{\prime}}=\frac{\left\langle P^{+}\right\rangle\left\langle P^{-}\right\rangle \delta_{m, 0}}{\left(\mathrm{i} \omega_{\nu}-J_{\nu}^{--}\right)\left(\mathrm{i} \omega_{\nu^{\prime}}-J_{\nu^{\prime}}^{--}\right)}-\frac{\left\langle P^{+}\right\rangle\left\langle P^{-}\right\rangle \delta_{\nu, \nu^{\prime}}}{\left(\mathrm{i} \omega_{\nu}-J_{\nu}^{--}\right)\left(\mathrm{i} \omega_{\nu+m}-J_{\nu+m}^{--}\right)}=\left(\Upsilon_{s}^{1}\right)^{--} \delta_{\nu \nu^{\prime}}-\left(\Upsilon_{\mathrm{d}}^{1}\right)^{--} \delta_{m 0}, \\
& +\bigcirc_{v+m}^{+v+\bar{m}_{++--}}=-G_{\nu}^{++} G_{\nu^{\prime}}^{--} \delta_{m, 0}=-\left(\Upsilon_{\mathrm{d}}^{1}\right)^{+-} \delta_{m 0}, \\
& -_{v+m}^{v+n^{+}+m_{--++}} v_{+}^{v_{+}}=-G_{\nu}^{--} G_{\nu^{\prime}}^{++} \delta_{m, 0}=-\left(\Upsilon_{\mathrm{d}}^{1}\right)^{-+} \delta_{m 0} \\
& -_{v+m}^{+v^{\prime}+m_{+-+-}} \bigcup_{v_{+}^{\prime}}^{v^{\prime}}=+G_{\nu}^{++} G_{\nu+m}^{--} \delta_{\nu, \nu^{\prime}}=\left(\Upsilon_{s}^{2}\right)^{++} \delta_{\nu \nu^{\prime}} \text {, }
\end{aligned}
$$

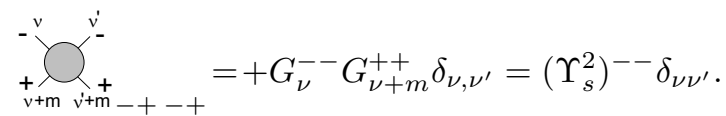

Three-time correlation function has the following non-zero components:

$$
\begin{aligned}
& \underset{\mathrm{m}}{+} \int_{\mathrm{v}+\stackrel{\mathrm{m}}{+++}_{+}^{+}}^{v_{+}}=-\frac{\left\langle P^{+}\right\rangle}{\left(\mathrm{i} \omega_{\nu}-J_{\nu}^{++}-U\right)\left(\mathrm{i} \omega_{\nu+m}-J_{\nu+m}^{++}-U\right)}+\frac{\left\langle P^{+}\right\rangle\left\langle P^{-}\right\rangle n_{+} \delta_{m, 0}}{\mathrm{i} \omega_{\nu}-J_{\nu}^{++}-U} \\
& \overline{\mathrm{m}} \int_{\nu+\overline{\mathrm{m}}_{---}}^{v}=-\frac{\left\langle P^{-}\right\rangle}{\left(i \omega_{\nu}-J_{\nu}^{--}\right)\left(\mathrm{i} \omega_{\nu+m}-J_{\nu+m}^{--}\right)}+\frac{\left\langle P^{+}\right\rangle\left\langle P^{-}\right\rangle n_{-} \delta_{m, 0}}{\mathrm{i} \omega_{\nu}-J_{\nu}^{--}}, \\
& \overline{\mathrm{m}} \int_{\mathrm{v}+\mathrm{m}_{-++}^{+}}^{+}=-n_{\mathrm{d}}^{-}\left\langle P^{-}\right\rangle G_{\nu}^{++} \delta_{m, 0} \\
& {\stackrel{\mathrm{m}}{\mathrm{m}+\overline{\mathrm{m}}_{+--}}}_{\mathrm{v}}^{\mathrm{v}}=-n_{\mathrm{d}}^{+}\left\langle P^{+}\right\rangle G_{\nu}^{--} \delta_{m, 0}
\end{aligned}
$$

All components of the two-time correlation function are non-zero:

$$
\begin{aligned}
& +\square+=-\sum_{\nu} \frac{\left\langle P^{+}\right\rangle}{\left(\mathrm{i} \omega_{\nu}-J_{\nu}^{++}-U\right)\left(\mathrm{i} \omega_{\nu+m}-J_{\nu+m}^{++}-U\right)}+\delta_{m, 0}\left\langle P^{+}\right\rangle\left\langle P^{+}\right\rangle n_{\mathrm{d}}^{+} n_{\mathrm{d}}^{+}, \\
& -\square-\sum_{\nu}=-\sum_{\left.P^{-}\right\rangle} \frac{\left\langle P^{-}\right\rangle}{\left(\mathrm{i} \omega_{\nu}-J_{\nu}^{--}\right)\left(\mathrm{i} \omega_{\nu+m}-J_{\nu+m}^{--}\right)}+\delta_{m, 0}\left\langle P^{-}\right\rangle\left\langle P^{-}\right\rangle n_{\mathrm{d}}^{-} n_{\mathrm{d}}^{-}, \\
& +\square-=-\delta_{m, 0}\left\langle P^{+}\right\rangle\left\langle P^{-}\right\rangle n_{\mathrm{d}}^{+} n_{\mathrm{d}}^{-}, \\
& -\square+{ }^{\mathrm{m}}+
\end{aligned}
$$




\section{References}

1. Falicov L.M., Kimball J.C., Phys. Rev. Lett., 1969, 22, 2267.

2. Freericks J. K., Zlatić V., Rev. Mod. Phys., 2003, 75, 1333.

3. Ramirez R., Falicov L. M., Phys. Rev. B, 1971, 3, 2425.

4. Lawrence J.M., Riseborough P.S., Parks R.D., Rep. Prog. Phys. 1981, 44, 1.

5. Kennedy T., Lieb E.H., Physica A, 1986, 138, 320.

6. Freericks J. K., Phys. Rev. B, 1993, 48, 14797

7. Shvaika A.M., Physica C, 2000, 341-348, 177; J. Phys. Studies, 2001, 5, 349.

8. Freericks J. K., Devereaux T.P., Phys. Rev. B, 2001, 64, 125110.

9. Shvaika A.M., Vorobyov O., Freericks J.K., Devereaux T.P., Phys. Rev. Lett., 2004, 93, 147402; Phys. Rev. B, 2005, 71, 045120

10. Brandt U., Mielsch C., Z. Phys. B: Condens. Matter, 1989, 75, 365; 1990, 79, 295;1991, 82, 37.

11. Georges A., Kotliar G., Krauth W., Rozenberg M., Rev. Mod. Phys., 1996, 68, 13.

12. Schubin S., Wonsowsky S., Proc. Roy. Soc., 1934, A145, 159

13. Hubbard J., Proc. R. Soc. London, 1963, Ser. A 276, 238.

14. Hirsch J. E., Physica C, 1989, 158, 236

15. Didukh, L.D., Sov. Phys. Solid State, 1977, 19, 711

16. Vollhardt D., Blümer N., Held K., Kollar M., Schlipf J., Ulmke M., Wahle J., Advances in Solid State Physics, 1999, 38, 383

17. Vollhardt D., Blümer N., Held K., Kollar M., Lecture Notes in Physics, 2001, 580, 191

18. Kollar M., Vollhardt D., Phys. Rev. B, 2001, 63, 045107

19. Appel J. et. al., Phys. Rev. B, 1993, 47, 2812.

20. Parr R. G. et. al., J. Chem. Phys., 1950, 18, 1561.

21. Schiller A., Phys. Rev. B, 1999, 60, 15660.

22. Shvaika A.M., Phys. Rev. B., 2003, 67, 075101;

23. Metzner W., Phys. Rev. B, 1991, 43, 8549.

24. Blackman A., Esterling D.M., Berk N.F., Phys. Rev. B, 1971, 4, 2412.

25. Koepernik K., Velický B., Hayn R., Eschrig H., Phys. Rev. B, 1998, 58, 6944.

26. Chung W., Freericks J.K., Phys. Rev. Lett., 2000, 84, 2461 


\title{
Динамічні сприйнятливості моделі Фалікова-Кімбала з корельованим переносом: загальний підхід
}

\author{
А.М.Швайка, О.Я.Фаренюк \\ Інститут фізики конденсованих систем НАН України, 79011 Львів, вул. Свєнціцького, 1 \\ Отримано 21 листопада 2008 р., в остаточному вигляді - 18 лютого 2009 р. \\ Досліджується модель Фалікова-Кімбала з корельованим переносом в границі безмежної розмір- \\ ності простору. Динамічні сприйнятливості розраховуються за допомогою теорії динамічного се- \\ реднього поля, що ґрунтується на розвиненнях за електронним переносом навколо одновузлової \\ границі. Досліджується чисельно частковий випадок напівеліптичної густини станів та діагонального \\ корельованого переносу та показано відсутність фазових переходів для всіх температур крім $T=0$, \\ що узгоджується з відомими результатами.
}

Ключові слова: модель Фалікова-Кімбала, корельований перенос, електронні сприйнятливості, теорія динамічного середнього поля, сильноскорельовані системи

PACS: $71.10 . F d$ 